\title{
Features and clinical significance of the ossification centres in the odontoid process based on micro-computed tomography
}

\author{
W. Wang 1, \#, X. Wang', \#, X. Ren ${ }^{3}$, Z. $\mathrm{Li}^{2}$, B. Su${ }^{4}, \mathrm{Y} . \mathrm{Xu}^{4}, \mathrm{X} . \mathrm{Xu}^{4}, \mathrm{D} . \mathrm{Lv}^{4}$, \\ W. Liư ${ }^{4}$, S. Zhang' ${ }^{2}$, L. Chen ${ }^{5}$, X. Li ${ }^{2}$ \\ 'Department of Emergency, Inner Mongolia People's Hospital, Hohhot, China \\ ${ }^{2}$ Human Anatomy Teaching and Research Section (Digital Medical Centre), Inner Mongolia Medical University, \\ Basic Medical College, Hohhot, China \\ ${ }^{3}$ Department of Endocrinology, Affiliated Hospital of Inner Mongolia Medical University, Hohhot, China \\ ${ }^{4}$ Graduate School, Inner Mongolia Medical University, Hohhot, China \\ ${ }^{5}$ Department of Haematology, Affiliated Hospital of Inner Mongolia Medical University, Hohhot, China
}

[Received: 19 November 2019; 21 November 2019]

Background: Microscopic structures of the ossification centres of the odontoid process were studied from the micro-computed tomography (CT) images of the axis, and the potential influence of the ossification centres with different microscopic structures on odontoid process fractures was analysed.

Materials and methods: Eighteen odontoid process specimens were randomly collected and scanned by micro-CT. The obtained images were then input into the software for further observation and measurement. Incomplete absorption of the ossification centres in the base was observed, along with the anatomic structure of the regions with incomplete ossification and structural parameters of the trabecular bones.

Results: The microscopic structures of the trabecular bones in the ossification centres in the base of the odontoid process could be clearly visualised from the micro-CT images. Among the 18 odontoid process specimens, 11 specimens were found with incomplete absorption of the ossification centres in the axis, the prevalence reaching up to $61 \%$. Regions with incomplete ossification varied in size and morphology, and their three-dimensional morphology was predominantly oval. Of all structural parameters examined for the trabecular bones, there were only significant differences in the degree of anisotropy between the regions with incomplete absorption of ossification centres and the average vertebral trabecular bones $(p<0.05)$.

Conclusions: Incomplete absorption of the ossification centres in the base of the odontoid process is a relatively prevalent condition. The cavitation effect of the trabecular bones may be the primary cause for odontoid process fractures. (Folia Morphol 2020; 79, 4: 845-850)

Key words: odontoid process, ossification centre, micro-computed tomography, cavitation effect

Address for correspondence: Dr. S. Zhang, Dr. L. Chen, Dr. X. Li, Human Anatomy Teaching and Research Section (Digital Medical Centre) Inner Mongolia Medical University, Basic Medical College, Hohhot, 010059, China; Department of Emergency, Inner Mongolia People's Hospital, Hohhot, 010017, China, tel: +86 471 6659007, fax: +86 471 6657562, e-mail: jay-8124@163.com; Ixchen499@126.com; 798242742@qq.com \#Wei Wang and Xing Wang contributed equally to this paper and share first authorship. 


\section{INTRODUCTION}

The odontoid process originates from the mesenchyme of the first cervical vertebra at the embryonic stage. During the growth and development of the odontoid process, the original two ossification centres gradually fuse into one. The epiphyseal plate of this fused ossification centre is located between the odontoid process and the axis vertebra. Under normal conditions, complete ossification occurs at about 6 years of age, leading to complete fusion between the odontoid process and the axis vertebra. However, residual ossification centres will appear if the ossification is incomplete, which unfortunately becomes an anatomical structure of mechanical weakness. This will further result in the fractures of the odontoid process of axis and hence the instability of the entire atlantoaxial complex and even the compression injury of the cervical spine. The pathogenesis of incomplete absorption of the ossification centres in the base of the odontoid process still remains unclear $[6,10$, $11,13]$. Most researches $[1,2,4]$ prefer the use of ordinary imaging techniques such as conventional computed tomography (CT), plain X-ray or magnetic resonance imaging (MRI) to characterise the absorption of the ossification centres in the axis. However, these imaging techniques can hardly visualise the absorption of the small trabecular bones in the ossification centres. Micro-computed tomography (micro-CT), known for its micro-grade $(\mu \mathrm{m})$ resolution, can clearly visualise the microscopic structure of the sample without destroying it. At present, micro-CT has been applied to the observation of the microscopic structure of the femoral trabecular bone [5, 7-9]. In the present study, micro-CT was used to visualise the absorption of the ossification centres of the axis, so as to further analyse the influence of the ossification centres in the base of the odontoid process on odontoid process fractures.

\section{MATERIALS AND METHODS}

\section{Baseline data}

According to the identification criteria of anthropology, 18 dry odontoid process specimens from adults (provided by the Teaching and Research Office of Human Anatomy at Inner Mongolia Medical University) were randomly selected. These specimens were aged between 45 and 63 years, with an average of 58.2. All specimens were of intact internal structure and no anatomical deformity by conventional X-ray, which satisfied the experimental requirements.

\section{Method}

Micro-CT scanner was used (Hiscan XM Micro CT, Suzhou Hiscan Information Technology Co., Ltd., Suzhou, China). After filtering and air calibration, the bone specimens were placed on the rotation stage and scanned segment by segment, in $25 \mu \mathrm{m}$ resolution. Uniform parameters were configured for the micro-CT scanner: voltage $60 \mathrm{kV}$, current $133 \mu \mathrm{A}$, single exposure time $50 \mathrm{~ms}$ and scan angle interval 0.5 degree.

The cross-sectional images of the bone specimens thus obtained were imported into the Hiscan Reconstruct software in the Digital Imaging and Communications in Medicine (DICOM) format. The segmental images of the bone specimens were integrated by using the image fusion technique in Hiscan Analyser software, and the intact micro-CT images of the axis specimens were obtained. These images contained all intact cancellous bone tissues of the axis, and the trabecular bones could be clearly visualised. Ossification centres in the base of the odontoid process and axis vertebrae were delineated as regions of interest (ROI) on the cross-sectional images using Hiscan Reconstruct software. The following parameter values of the trabecular bones were calculated using the built-in software: bone volume fraction $(\mathrm{BV} / \mathrm{TV})$, bone surface/bone volume (BS/BV), bone surface density (BS/TV), trabecular thickness (Tb.Th), trabecular number (Tb.N), trabecular spacing (Tb.Sp), degree of anisotropy (DA), and bone density [14]. The parameters were analysed statistically.

\section{Statistical method}

The data were input into Excel and SPSS17.0 for sorting and statistical analysis. The data were expressed as mean \pm standard deviation $(x \pm s)$. Non-parametric test was performed at the significance level of $\alpha=0.05$. $\mathrm{P}<0.05$ was taken to indicate significant difference.

\section{RESULTS}

Clear images of the overall structure of the axis were obtained by the micro-CT scan, and the trabecular bones were clearly visible for each vertebra. Of the 18 specimens of the axis, 11 specimens were found with incomplete absorption of the ossification centres in the base of the odontoid process, accounting for about $61 \%$. As a result of incomplete ossification, cavities were formed and the connections between the trabecular bones were easily differentiable. That 


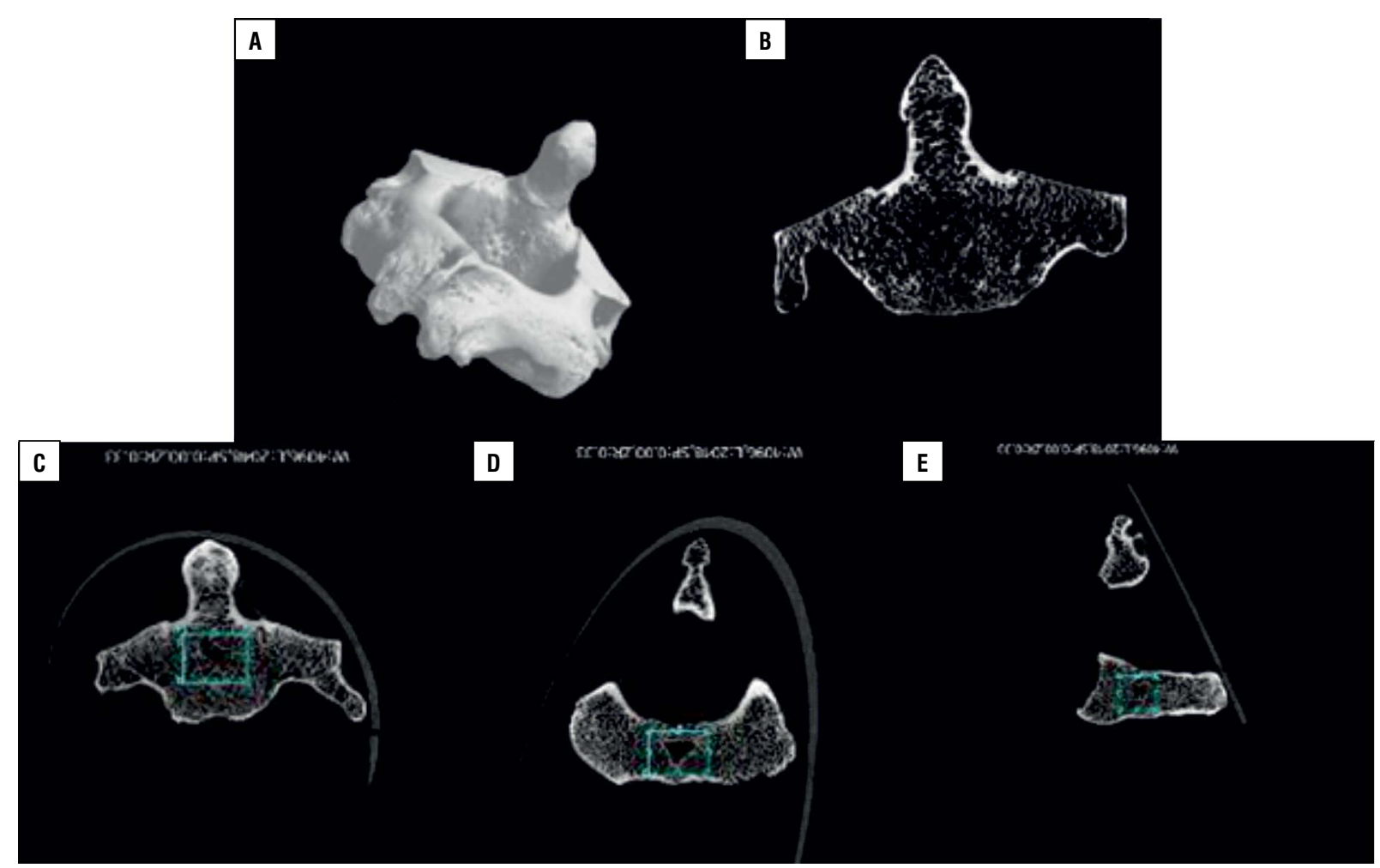

Figure 1. A, B. Coronal micro-computed tomography angiography images showing no unclosed ossification centres in the base of the odontoid process; C-E. Respectively sagittal, coronal and horizontal images showing unclosed ossification centres in the base of the odontoid process.

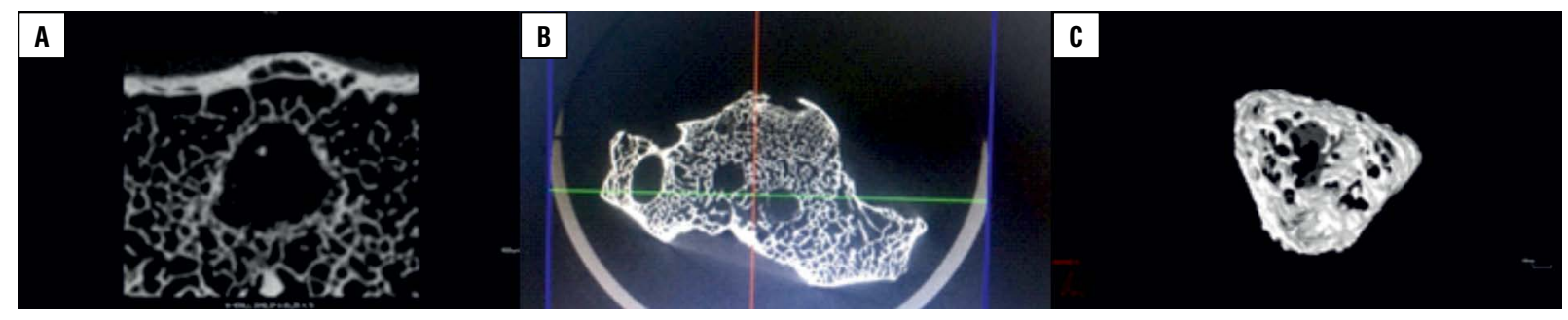

Figure 2. A-C. Each structure of the unclosed ossification centres in the base of the odontoid process and the micro-computed tomography image after three-dimensional reconstruction.

is, the ossification centres were continuous with the trabecular bones of other vertebrae. The internal margins of the cavities were presented as smooth and regular calcification shadows and were hyperintense on the micro-CT images.

Regions with incomplete ossification varied in size and morphology, and their three-dimensional (3D) morphology was predominantly oval. Some of them were of triangular pyramidal shape or flat oval shape. There was even one case with double oval shape in the axis. These regions were usually of a small size in 3D view. The transverse and vertical diameters of the maximum cavities measured on the coronal plane accounted for less than $10 \%$ of the transverse and vertical diameters in the same planar vertebral structure, respectively. Such observations occurred in 8 patients, accounting for about $80 \%$. Larger cavities were sometimes formed in the regions of incomplete ossification. For these cavities, the transverse and vertical diameters measured on the coronal plane accounted for about $30 \%$ of the transverse and vertical diameters in the same planar vertebral structure, respectively. Such observations occurred in 3 patients, accounting for about $20 \%$ (Figs. 1, 2).

Regions of interests were selected from the micro-CT images of incomplete ossification in the base 
Table 1. Comparison of trabecular bone parameter values at different sites of the axis $(x \pm s, n=11)$

\begin{tabular}{lccccccc}
\hline & BS/BV [\%] & BV/TV [\%] & BS/TV [\%] & Th.Th [mm] & Th.Sp [mm] & Tb.N [mm-1] & DA \\
\hline Overall axis vertebrae & $15.99 \pm 6.23$ & $0.43 \pm 0.06$ & $6.77 \pm 2.71$ & $0.13 \pm 0.01$ & $0.30 \pm 0.07$ & $2.37 \pm 0.46$ & $0.33 \pm 0.31$ \\
Regions with incomplete ossification & $17.53 \pm 10.24$ & $0.40 \pm 0.14$ & $7.94 \pm 7.42$ & $0.14 \pm 0.01$ & $0.36 \pm 0.13$ & $2.12 \pm 0.48$ & $0.64 \pm 0.23^{*}$ \\
\hline
\end{tabular}

${ }^{*} \mathrm{P}<0.05$ as compared with the incomplete ossification group; abbreviations - see text

of the odontoid process in 11 specimens. Calculations showed that except for DA $(p<0.05)$, no significant differences were found between the following parameters of the trabecular bones in ROIs with incomplete ossification and the overall values of the vertebral trabecular bones: BV/TV, BS/BV, BS/TV, Tb.Th, Tb.N, Tb.Sp and BD ( $p>0.05)$ (Table 1).

\section{DISCUSSION}

Ossification centres of the axis in human usually fuse into trabecular bones before the age of 6 . The trabecular bones formed after complete absorption of the ossification centres are uniform and consistent as those in other vertebrae. Since fusion of the ossification centres is already complete after reaching adulthood without any traces of ossification, no regions of mechanical weakness will be found in the normal axis vertebrae. However, cavities will be formed instead if the ossification is incomplete for some reasons and become the sites of mechanical weakness, leading to odontoid process fractures. The pathogenesis of incomplete ossification still remains unclear. Aydin and Cokluk [2] studied fusion of the ossification centres in the cartilage of the base of odontoid process in children by using MRI. They found that the true lower bound of the odontoid process should be located in the ossification centres in the cartilage in the central base of odontoid process. We also found by using the micro-CT scan of the entire axis that the ossification centres were all below the line connecting the highest points on the bilateral superior articular surfaces of the axis. The ossification centres were connected to the trabecular bone at the inferior end of the odontoid process via the trabecular bone. Anatomically, this is considered the lower margin of the base.

In the present study, the formation of cavities due to incomplete ossification of the axis vertebrae was a common condition, and these cavities varied in size and morphology. Given the significant cavitation effect, these cavities are considered to be of greatly importance for understanding the mechanism and for the classification of odontoid process fractures.
Recent years have witnessed a progress in high-resolution imaging techniques, especially micro-CT scan, with a reduction in cost. This provides a better pathway to studying the mechanism of odontoid process fractures and towards a refined classification of odontoid process fractures. In normal axis vertebrae, the rod-like trabecular bones in the ossification centres form a complex network structure through the transverse or oblique connections with trabecular bones in other vertebrae. Energy from the stressed axis can be transmitted along the trabecular bones to other sites of the axis. In other words, the atlantoaxial complex can stably transmit the energy through the entire axial bone. However, the cavitation effect induced by incomplete ossification of the trabecular bones has an adverse impact on energy transmission. Anderson and D'Alonzo [1], using the conventional X-ray, believed that type II and III odontoid process fractures occurred in the base. In the type III fracture, the fracture line extended to the axis vertebrae. However, Anderson's classification system can only roughly analyse the axis fractures, but may fail to accurately differentiate between different types of odontoid process fractures. Grauer et al. [4] provided an accurate analysis of the distribution of the fracture line on the odontoid process: the fracture line extended from deep inside the vertebra to the outside, and the classification of the fractures varied with or without the involvement of the superior articular surfaces of the axis. We also believe that if an ossification centre with incomplete absorption is close to the line connecting the highest points on the bilateral superior articular surfaces of the axis, it will be easily affected by the energy transmitted to this position, leading to fractures. If an ossification centre with incomplete absorption is farther away from the line connecting the highest points on the bilateral superior articular surfaces of the axis, the energy will be dissipated by other trabecular bones of the axis vertebrae, which makes the fractures unlikely.

In our study, the size and morphology of the ossification centres with incomplete absorption should 


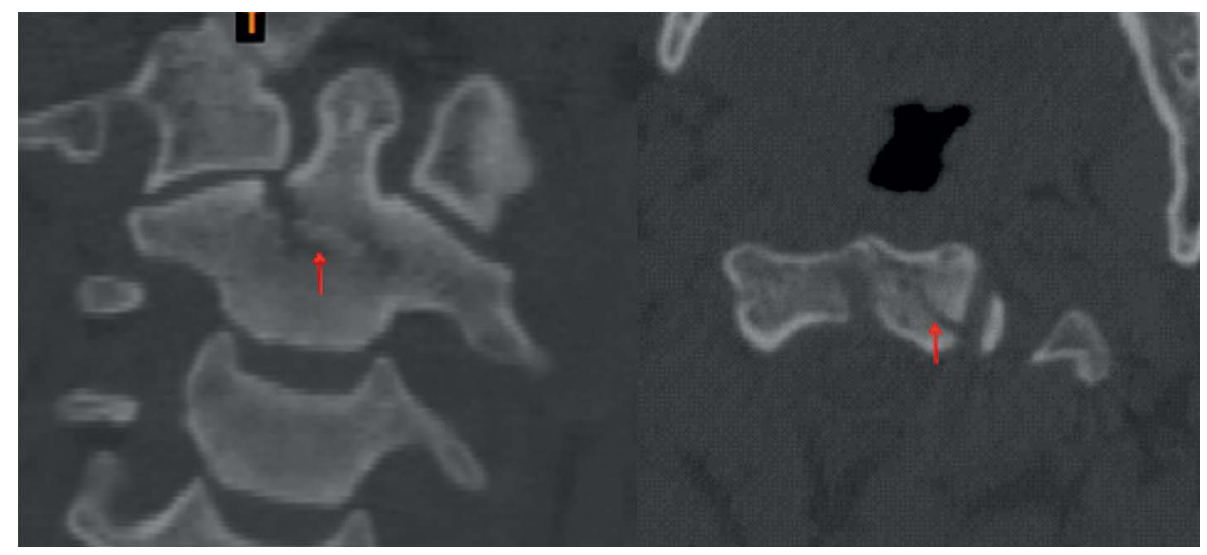

Figure 3. Type III fracture in the unclosed ossification centres in the base of the odontoid process (red arrowheads).

be closely associated with the mechanism and classification of the odontoid process fractures. The larger the size of the ossification centres with incomplete absorption, the more significant the cavitation effect would be, and also the greater the potential influence on the fractures in the base of the odontoid process. When the odontoid process is under the stress imposed by the head from different directions, the energy will be transmitted to the inside of the axis vertebrae via the trabecular bones in the odontoid process. If, in the 3D view, the cavities in the unclosed ossification centres account for a smaller proportion of the total volume of the vertebra, the energy transmitted to the unclosed ossification centres will not be large enough to tear the mechanically weak trabecular bones on the medial side. Rather, the energy will be transmitted to the surrounding along the normal trabecular bones. But if the cavities are sufficiently large, the cavitation effect will be much greater. As a result, when the energy is transmitted to the cavities in the unclosed ossification centres, the mechanically weak trabecular bones on the medial side may be first torn. After that, the energy will be transmitted to different directions along the torn trabecular bones, and the vertebral bodies of the axis will be fractured, probably with the involvement of unilateral or bilateral articular surfaces of the axis. This may be the possible mechanism of the occurrence of odontoid process fractures in clinic [3, 12, 15] (Fig. 3).

\section{CONCLUSIONS}

After delineating the trabecular bones in the ROls with incomplete ossification, relevant parameters were calculated for the trabecular bones. Then a comparison was made with the average parameter values of the vertebral trabecular bones. We found that except for DA, there were no significant differences in other parameters between the two groups of trabecular bones. By combining with the analysis of the micro-CT images, we can reasonably believe that significant ossification and absorption effect exists on the medial margin of the cavities in the ossification centres in the base of the odontoid process where the ossification is incomplete. Taken together, our results shed new light on the imaging diagnosis of odontoid process fractures. If there are hyperintense shadows upon conventional CT scan, cavities with incomplete ossification can be considered.

\section{Acknowledgements}

This study was supported by Study on the Natural Science Foundation of China $(81660358,81860382$, $81860383,81560348)$, the Inner Mongolia Medical University Science and Technology Million Project (YKD2015KJBW003, YKD2017KJBW(LH)062), Inner Mongolia Autonomous Region Health and Family Planning Commission Medical and Health Planning Research Project (201703015), Foundation of Inner Mongolia People's Hospital (201824), Nature Science Foundation of Inner Mongolia Autonomous Region of China (2017MS(LH)0835, 2019LH08003), and Research Project Plan of Affiliated Hospital of Inner Mongolia Medical University (NYFY YB 004).

\section{REFERENCES}

1. Anderson LD, D'Alonzo RT. Fractures of the odontoid process of the axis. J Bone Joint Surg Am. 2004; 86(9): 2081, doi: 10.2106/00004623-200409000-00032.

2. Aydin $K$, Cokluk $C$. The segments and the inferior boundaries of the odontoid process of $\mathrm{C} 2$ based on the magnetic resonance imaging study. Turk Neurosurg. 2008; 18(1): 23-29, indexed in Pubmed: 18382973. 
3. Badshah $M$, Soames $R$, Ibrahim $M$, et al. Surface anatomy of major anatomical landmarks of the neck in an adult population: A Ct Evaluation of Vertebral Level. Clin Anat. 2017; 30(6): 781-787, doi: 10.1002/ca.22907, indexed in Pubmed: 28514499.

4. Grauer JN, Shafi B, Hilibrand AS, et al. Proposal of a modified, treatment-oriented classification of odontoid fractures. Spine J. 2005; 5(2): 123-129, doi: 10.1016/j. spinee.2004.09.014, indexed in Pubmed: 15749611.

5. Markvart M, Darvann TA, Larsen $P$, et al. Micro-CT analyses of apical enlargement and molar root canal complexity. Int Endod J. 2012; 45(3): 273-281, doi: 10.1111/j.13652591.2011.01972.x, indexed in Pubmed: 22044111.

6. Megan GE, Michael K. Fractures of the axis: a review of pediatric, adult, and geriatric injuries. Curr Rev Musculoskelet Med. 2016; 9(4): 505-512, doi: 10.1007/s12178016-9368-1, indexed in Pubmed: 27686572.

7. Perilli E, Parkinson IH, Reynolds KJ. Micro-CT examination of human bone: from biopsies towards the entire organ. Ann Ist Super Sanita. 2012; 48(1): 75-82, doi: 10.4415/ ANN_12_01_13, indexed in Pubmed: 22456020.

8. Peyrin F. Evaluation of bone scaffolds by micro-CT. Osteoporos Int. 2011; 22(6): 2043-2048, doi: 10.1007/s00198011-1609-y, indexed in Pubmed: 21523402.

9. Ritman EL. Current status of developments and applications of micro-CT. Annu Rev Biomed Eng. 2011; 13: 531-552, doi: 10.1146/annurev-bioeng-071910-124717, indexed in Pubmed: 21756145.
10. Shoja MM, Johal J, Oakes WJ, et al. Embryology and pathophysiology of the Chiari I and II malformations: A comprehensive review. Clin Anat. 2018; 31(2): 202-215, doi: 10.1002/ca.22939, indexed in Pubmed: 28612426.

11. Shoja MM, Ramdhan R, Jensen CJ, et al. Embryology of the craniocervical junction and posterior cranial fossa, part I: Development of the upper vertebrae and skull. Clin Anat. 2018; 31(4): 466-487, doi: 10.1002/ca.23049, indexed in Pubmed: 29345006.

12. Shoja MM, Ramdhan R, Jensen CJ, et al. Embryology of the craniocervical junction and posterior cranial fossa, part II: Embryogenesis of the hindbrain. Clin Anat. 2018; 31(4): 488-500, doi: 10.1002/ca.23048, indexed in Pubmed: 29344994.

13. Smith HE, Kerr SM, Fehlings MG, et al. Trends in epidemiology and management of type II odontoid fractures: 20-year experience at a model system spine injury tertiary referral center. J Spinal Disord Tech. 2010; 23(8): 501-505, doi: 10.1097/BSD.0b013e3181cc43c7, indexed in Pubmed: 20940632.

14. Tassani S, Perilli E. On local micro-architecture analysis of trabecular bone in three dimensions. Int Orthop. 2013; 37(8): 1645-1646, doi: 10.1007/s00264-013-1989-z, indexed in Pubmed: 23835557.

15. Watanabe M, Sakai D, Yamamoto Y, et al. Analysis of predisposing factors in elderly people with type II odontoid fracture. Spine J. 2014; 14(6): 861-866, doi: 10.1016/j. spinee.2013.07.434, indexed in Pubmed: 24055610. 